\title{
Conclusión \\ Propuestas para construir un modelo de gestión de los cuidados de larga duración basado en la equidad social, la igualdad de género y la cohesión territorial
}

\author{
Raquel Martínez Buján \\ Universidade da Coruña

\section{Lucía Martínez Virto} \\ Universidad Pública de Navarra \\ <lucia.martinez@unavarra.es>
}

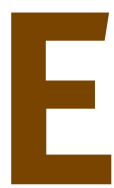

n el despliegue de cada uno de los artículos de este número especial sobre La organización social de los cuidados de larga duración en un contexto de crisis y de precariedad laboral se han analizado los cambios que la provisión del trabajo de cuidados ha experimentado en las últimas décadas. Por ello, los documentos incluidos dividen su atención en los nuevos derroteros de las directrices de la política estatal en el ámbito de la atención personal y su diversidad en la implementación a escala regional, en las modificaciones en la distribución de las tareas de cuidar en el interior de las familias, así como en la cada vez mayor presencia del mercado privado en la externalización de estas actividades (especialmente en forma de servicio doméstico). A partir de la información que proporcionan cada una de las contribuciones, presentamos una serie de reflexiones, propuestas o estrategias que podrían seguirse para desarrollar un modelo de gestión de los cuidados de larga duración que permita su distribución acorde a las necesidades de las personas dependientes y sus cuidadores familiares, y que se adecue a las fórmulas de privatización seguidas en este ámbito. En concreto, aglutinamos estas estrategias en tres ejes de actuación que pasamos a comentar a continuación.

\section{Promover un modelo social que considere los cuidados como aspecto central}

Es necesario crear una estructura laboral, económica y política que permita mantener la responsabilidad de la política pública en este ámbito, pero que también incluya y reconozca la participación de las familias en la provisión. Las intervenciones deben ir dirigidas a fomentar un mercado de trabajo que sea acorde a las necesidades de las familias y de los trabajadores/as, superando las contradicciones entre los tiempos de trabajo y los tiempos de reproducción de la vida. Al mismo tiempo, la política pública debe encaminarse a potenciar medidas que no redunden en delegar la carga de los cuidados dentro de las familias en las mujeres; las medidas que se implementen deben estar a la altura de las expectativas sociales y profesionales de las nuevas generaciones y no pueden sostenerse en la sobrecarga a las mujeres de mediana edad con las actividades de cuidados. Ya se ha demostrado en los documentos que se incluyen en este monográfico que algunas recientes directrices, como las prestaciones económicas de cuidados en el entorno familiar, no suponen en todos los casos una ayuda monetaria que solventa la carga de la asistencia en los domicilios, sino que fomentan precisamente que las cuidadoras de la parentela sigan ejerciendo estas actividades. En este sentido, las políticas públicas de actuación en el marco de los cuidados de larga duración deberían tender a incluir una perspectiva de género y generacional que evite el encapsulamiento de ciertos grupos sociales (definidos por su sexo y edad) en esta ocupación. 


\section{Vincular políticas de empleo activo con el sector de cuidados a la dependencia}

La privatización del trabajo familiar de cuidados en España ha sido una de las principales modificaciones que ha experimentado la organización social de los cuidados en nuestro país. En las últimas dos décadas, se ha incrementado el número de hogares que contratan a empleadas de hogar para cumplir con estas tareas y, de hecho, los análisis académicos redundan en afirmar que esta actividad se convirtió en el sector de actividad principal de las mujeres migrantes procedentes de América Latina. Así, pioneras corrientes teóricas sobre este ámbito, como ya la mundialmente reconocida 'teoría del trabajo social', han coincidido en caracterizar el régimen de cuidados en España como el de 'una migrante en la familia'. Si la familia no puede o no quiere desempeñar las tareas de atención personal, se busca la opción de dispensación de los cuidados más similar a los que puede realizar la familia, es decir, aquellos que se caracterizan por una elevada carga afectiva y que no están profesionalizados. La contratación de empleadas de hogar alivia las tensiones que el trabajo de asistencia genera dentro del grupo de parentesco, sin perder la ideología dominante de que deben ser los parientes los que se encarguen de su gestión. Además, la figura de la cuidadora privada permite a la persona mayor envejecer dentro de su entorno.

No debemos obviar que esta privatización de los cuidados en torno al servicio doméstico se produce principalmente en aquellos países con una cultura de cuidados más centrada en la familia y con una escasez en la cobertura de los servicios sociales públicos. Que sean mujeres migrantes latinoamericanas las trabajadoras sobre las que recae esta actividad ya depende del modelo migratorio y de cómo se estructura el mercado laboral en este sector. En el caso español, la acelerada llegada de población migrante irregular, sobre todo procedente de América Latina en el período 2003-2007, donde los flujos migratorios fueron eminentemente femeninos, junto con una desregulación en las ocupaciones relacionadas con los cuidados a domicilio, propiciaron la absorción de estas mujeres en el servicio doméstico. Los estudios cualitativos que han analizado la incidencia del fenómeno coinciden en afirmar que la repercusión de este empleo sobre la población extranjera femenina ha sido tan elevado que el trabajo de cuidados no profesional ha sido su puerta de entrada al mercado laboral español.

No obstante, la concentración de mujeres inmigrantes en este sector de cuidados no está relacionada únicamente con el crecimiento de la demanda de asistencia, ni tampoco con las limitaciones del sistema de protección social formal. Dos elementos más influyen en el auge de este proceso. Por una parte, los vínculos culturales y las preferencias de los/as empleadores/as en cuanto a la procedencia étnica de las cuidadoras también determinan la composición de la mano de obra. Existe una tendencia generalizada entre las familias españolas a considerar a las mujeres latinoamericanas como más ‘cariñosas' y 'pacientes', lo que las convierte en las preferidas para desempeñar estas actividades. Por otra parte, la extensión de políticas públicas dirigidas a los cuidados de larga duración basadas en transferencias económicas, más que en servicios sociales, ha sido otro de los factores clave en la consolidación de la mercantilización.

Estas prestaciones monetarias han sido muy desarrolladas por la Ley de Promoción de Promoción de la Autonomía Personal y Atención a las Personas en Situación de Dependencia (LAPAD), al menos antes de la introducción de las políticas de austeridad. Fueron concebidas como una especie de subsidios que se otorgan directamente a las familias bien para que puedan comprar en el mercado privado el servicio social que el sistema haya valorado oportuno, bien para compensar a la cuidadora familiar dentro del hogar que realiza las tareas asistenciales. Cabe mencionar que, aunque en los últimos tres años las comunidades autónomas tienden a limitar la concesión de estas prestaciones, la prestación económica para cuidados en el entorno familiar fue, desde los inicios de la LAPAD, el recurso más desarrollado. Algunas investigaciones centradas en evaluar los efectos de esta Ley de Dependencia indican que los salarios concedidos a los cuidados familiares ya se están utilizando para remunerar a cuidadoras privadas no regulares contratadas mediante el servicio doméstico. El caso de España es especialmente alarmante, puesto que la Ley no contempla la inversión de dichos subsidios en la contratación de cuidadoras no profesionales (tal y como sucede en otros países, como Alemania y Austria, por ejemplo), no se ha adoptado una categoría laboral específica para este figura y además se han otorgado sin realizar ningún seguimiento sobre cómo se emplea el dinero recibido. Esta desregulación del programa de transferencias monetarias influye en las condiciones en las que las trabajadoras entran en el sector de cuidados. Por un lado, al no poder contratarse legalmente a cuidadoras en el hogar a través de esa subvención, las limita a concentrarse en la vía del servicio doméstico irregulary, en consecuencia, en los empleos con las condiciones más precarias. Por otro lado, mitiga su acceso al sector formal de cuidados.

A partir de los artículos incluidos en este monográfico, consideramos esencial regular este sector de asistencia personal mediante la interrelación de las políticas públicas en tres vertientes: las políticas de la protección social a la dependencia, las políticas de empleo y las políticas de extranjería. La propuesta que recomendamos es la de subvencionar la privatización de los cuidados a través de los programas de protección social, garantizando un marco de condiciones laborales y salariales para estas empleadas que se asimile al del resto de los trabajadores. La finalidad de 
estas acciones es evitar la creación de mercados de cuidados sumergidos que afectan a la integración social de las trabajadoras y a la calidad de la atención suministrada. La implementación de estas medidas, sin duda, deberá acompañarse de la creación de convenios bilaterales y de cursos de formación específicos que capaciten a las futuras trabajadoras en estas actividades. Los beneficios son indudables: se mitiga la extensión de la red de empleo informal en esta actividad, se potencia la llegada de flujos migratorios regulares y además permite desarrollar las capacidades formativas de las empleadas. Igualmente cabe destacar el potencial que este sector ofrece para la creación de empleo. Las condiciones básicas para la implementación de esta propuesta serían:

- Reconocer la figura de la cuidadora no profesional a domicilio e incluirla como medio de provisión en los programas públicos de apoyo a las situaciones de dependencia. De esta manera se podrían enlazar las políticas públicas de protección social con las políticas migratorias y de empleo.

- Clasificar los cuidados en hogares privados en un estatus laboral alternativo al del servicio doméstico, de manera que pueda tenderse hacia una profesionalización de la provisión de cuidados en los hogares privados.

- Potenciar las formaciones especializadas dirigidas a la asistencia de personas con diversidad funcional. Conocer los problemas médicos asociados a ciertas enfermedades y fomentar las capacidades emocionales para atender a personas que requieren cuidados paliativos.

- Establecer convenios colectivos con aquellos países con los que se han establecido circuitos migratorios femeninos, fomentando la contratación en origen de cuidadoras y la implantación de actividades formativas en este ámbito.

\section{Afinar las fórmulas de administrar los recursos ofrecidos por la Ley de Dependencia y unificar los criterios de su financiación}

La existencia de un soporte financiero sólido es, sin duda, determinante para garantizar que una norma pueda desarrollarse y tener un impacto social reseñable. En el caso de la LAPAD, la ausencia de una reserva adecuada de capital que permitiese desarrollar sus servicios y prestaciones ha sido uno de sus principales frenos. Además, la norma, de partida, contaba con ciertas limitaciones que marcaron la posibilidad de que su desarrollo fuera sustancialmente diverso entre comunidades autónomas y alarmantemente desigual entre beneficiarios/as.

En cuanto a la diversidad territorial de modelos y al impacto de la norma, debe señalarse, en primer lugar, que su gestión recayó fundamentalmente en los servicios sociales, un sistema cuyo desarrollo es competencia de administraciones autonómicas y locales, por lo que, de partida, las formas de gestión se caracterizaron por la diversidad territorial. En segundo lugar, la financiación se estableció a partir de dos niveles. El primero de ellos un nivel mínimo acordado y definido a partir del número, grado de dependencia y tipología de prestaciones de las personas beneficiarias. Este primer nivel era financiado por la Administración central. En segundo lugar, un nivel acordado entre la Administración General del Estado y las comunidades autónomas; y en tercer lugar, un nivel adicional de protección establecido por las comunidades autónomas. Por tanto, a partir de estos niveles de protección, la financiación de las prestaciones y servicios a la dependencia se definía en función del tipo de prestación reconocida y gestionada por la comunidad autónoma, del número de beneficiarios, de su grado y nivel reconocido, del acuerdo entre la Administración General del Estado y las comunidades autónomas, y del retorno del copago de los usuarios. A partir de aquí, se fue construyendo un mapa de atención a la dependencia basado no solo en las diversas formas de gestión entre territorios, sino en la desigual distribución de los recursos entre comunidades autónomas.

Igualmente, la distribución de los servicios y prestaciones estableció importantes desigualdades entre las personas en situación de dependencia. La tercera 'pata' sobre la cual se asienta en sistema español de atención a la dependencia son las aportaciones de los/as beneficiarios/as. El copago de los servicios de atención a la dependencia depende, fundamentalmente, del modelo aplicado, de las rentas de los/as beneficiarias/as (sin patrimonio) y de los precios públicos definidos para cada servicio. No obstante, la aportación de los usuarios/as tiene un techo que provoca que aquellas rentas más altas se beneficien de una reducción del precio de sus servicios, mientras que para las rentas bajas el acceso a los servicios supone un mayor peso en su presupuesto. Por tanto, la ausencia de equidad en términos de territoriales también se produce en términos de clase social o rentas.

Todos estos elementos que, de partida, ponían en cuestión la universalidad de la LAPAD se vieron intensificados con el cambio de Gobierno. Además de a la crisis económica que atravesaba España, ello se debió, fundamentalmente, a que la Ley no contaba con un consenso sólido que garantizara su desarrollo al margen de la etapa política del Estado. Este cambio de rumbo se materializó en el Real Decreto Ley $20 / 2012$, donde el sistema de protección a la dependencia fue uno de los más castigados.

Las medidas de austeridad implementadas en materia de dependencia marcaron la consolidación de la Ley, el papel de los actores, la precariedad del sector y la desigualdad entre personas en situación de dependencia. Tal y como ha podido comprobarse 
lo largo del monográfico, las medidas definidas en el Decreto buscaban reducir el coste económico del sistema de atención a la dependencia a partir de una serie de medidas que ensancharon la brecha de desigualdad en el acceso a prestaciones y servicios:

- El primer bloque de recortes que redujo el coste de la dependencia se sustentó en la modificación de los grados de dependencia, en la eliminación de los niveles de intensidad vinculados a cada grado, en el retraso del acceso a las personas con dependencia de grado moderado al sistema, en la implementación de algunas incompatibilidades entre servicios y en el aumento de los copagos. Por otro lado, la reducción de la financiación de la Administración General del Estado también se debió a un recorte de la aportación estatal a las comunidades autónomas por la disminución de las cuantías de nivel mínimo y la supresión del nivel acordado. Por último, esta limitación fondos se definió igualmente por una menor exigencia a las comunidades autónomas en el nivel mínimo, que se materializó en una reducción de la intensidad de las prestaciones y los servicios.

- En segundo lugar, estas medidas provocaron que el sistema público de la dependencia fuera todavía menos equitativo por la excesiva presión de los copagos en muchas prestaciones. El aumento del copago implicó nuevos riesgos para los hogares que, además de tener personas dependientes, estaban afectados directamente por el desempleo y la pobreza, debido a que medidas de ajuste aparentemente universales, como el copago, suponen un mayor peso en los ya débiles presupuestos de muchas familias. Por ello, el aumento de las tasas ha empujado a muchas familias a reducir, de manera voluntaria, los servicios que se prestaban a la persona beneficiaria, o incluso prescindir de ellos, provocando la "autoexclusión" deliberada del sistema en las personas con menos recursos económicos.

- En tercer lugar, el Real Decreto Ley 20/2012 también modificó la capacidad de acción de los agentes implicados en la atención a la dependencia. Por un lado, refundación de la LAPAD tenía entre sus objetivos aumentar la oportunidad de negocio en el sector de la dependencia. Por otro lado, la reforma modificó la prestación económica de cuidados en el entorno familiar (PECEF) y eliminó la bonificación de la cuota de la Seguridad Social para las personas cuidadoras familiares. A partir de estos cambios, los cuidados familiares retornan al espacio invisible y aumentan las prestaciones vinculadas a servicios, en contraposición con las de entorno familiar. La apuesta por el apoyo público para el acceso a servicios ha ido de la mano de un aumento de la mercantilización del sector.

- Por último, la implementación de los cambios que definía la norma alteró y complejizó de manera sustancial la gestión de la LAPAD, debido a la falta de información ágil y adecuada ofrecida a los profesionales encargados de su gestión. Este hecho provocó grandes conflictos a la hora de gestionar la Ley, debido a que, en ocasiones, la información ofrecida en prensa y la que disponían los profesionales era contradictoria.

En este escenario, nos encontramos ante la necesidad de redefinir las fórmulas de financiación y gestión de la LAPAD, de tal forma que puedan agilizarse los pagos, garantizarse mecanismos equitativos en el acceso a servicios, desbloquearse los procedimientos de resolución y reducirse las listas de espera. Sin embargo, estas cuestiones no son las únicas disfuncionalidades del sistema. Desgraciadamente, hemos visto como la universalidad con la que partía la norma en 2006 se ha ido reduciendo, provocando que las familias con presupuestos más débiles no puedan acceder a muchos servicios o que las comunidades autónomas con menor capacidad de gestión reciban una menor financiación del sistema. En este sentido, sería esencial mejorar la coordinación multinivel, para que el mapa de recursos y prestaciones no se adapte únicamente al tipo de prioridades políticas de cada región, sino también a las necesidades de las personas con dependencia y sus cuidadores/as.

En definitiva, a la luz de las reflexiones presentadas en este monográfico consideramos fundamental, en el plano territorial, unificar las vías de financiación del sistema de dependencia, de tal forma que seamos capaces de evolucionar hacia un sistema de provisión de recursos equitativo. Por otro lado, en el ámbito social, es necesario agilizar la gestión y potenciar la atención urgente a aquellos hogares que se encuentren en situación de emergencia social por tener, por ejemplo, varias personas dependientes en el domicilio, así como promover que aquellos hogares en situación de emergencia económica no se vean privados de recursos de cuidado por razones económicas. Igualmente, las reflexiones ofrecidas ponen sobre la mesa la necesidad inevitable de vincular el modelo de atención a los cuidados tanto con el mercado de trabajo como con las políticas de extranjería, debido a que el carácter subsidiario del que parten las actuales políticas de protección social a la dependencia hace necesario revisar los efectos sociales que generan en términos de sexo, edad y origen étnico. 